\title{
A Novel Memory Subsystem and Computational Model for Parallel Reconfigurable Architectures
}

\author{
Yamuna Rajasekhar and Ron Sass \\ Reconfigurable Computing Systems Laboratory \\ University of North Carolina at Charlotte \\ \{yrajasek,rsass\}@uncc.edu
}

\begin{abstract}
While FPGA and other reconfigurable technologies have dramatically increased in size and speed, memory technology has had only modest improvements. Relative to logic speeds, memory latency is virtually flat and physical constraints on external pins limit memory bandwidth. Unfortunately, the traditional cache hierarchy found in fixedfunction integrated circuits has evolved to support sequential processors and is ineffective for highly parallel architectures.

This paper proposes a novel memory subsystem and computational model for reconfigurable architectures. It envisions a system where computational cores are oversubscribed with atomic tasks and the memory subsystem enables (1) hiding of latency by enabling the cores to overlap computation and memory transactions and (2) the system to fully utilize the available memory bandwidth. The first step in this grand vision is to change the memory model. Instead of a byte-addressable, global address space, a named segment memory controller is introduced and an FPGA-based implementation presented in this paper.
\end{abstract}

\section{Introduction}

Steady increases in the density of Integrated Circuits (IC) has resulted in more transistors per chip and faster transistors. Unfortunately, memory technology has not seen similar improvements. When measured in terms of system clock cycles, memory latency has grown dramatically and is expected to approach 1,000 clock cycles to access off-chip DRAM in the next decade 8. Moreover, it is highly likely that the long-term trend in memory latency, illustrated in Figure 1, will continue for the next decade. While this so called "Memory Wall" was recognized in the mid-1990s 10 chip architects have been able to mitigate the latency issue for single core microprocessors through the extensive use of caches and cache hierarchies. But, this approach has come with a high cost. It requires a substantial amount of resources (from $50 \%$ to $90 \%$ of the transistors on a chip) 6] and it consumes a significant amount of energy to operate [1. Furthermore, to maximize performance for a specific application (by increasing cache line locality in the hierarchy) requires an enormous effort, to the point that humans typically achieve a tiny fraction of the theoretical performance and instead rely on automated performance tuning [3, 5, 7,.

D. an Mey et al. (Eds.): Euro-Par 2013 Workshops, LNCS 8374, pp. 444-453, 2014.

(C) Springer-Verlag Berlin Heidelberg 2014 


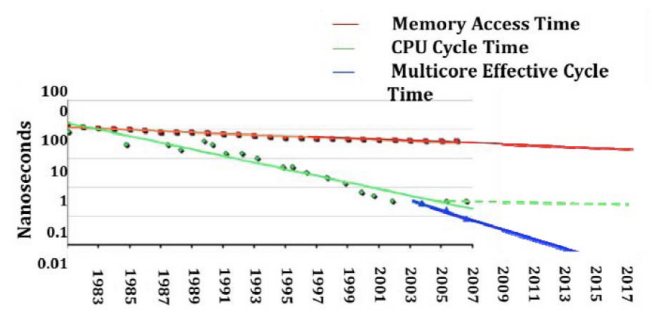

Fig. 1. Memory latency compared to (processor) clock frequency

Further complicating the memory problem facing modern parallel systems is memory bandwidth. First, the traditional cache hierarchy is optimized for single core processor performance. It devalues memory bandwidth in favor of statistically improving the memory latency observed by the processor. From the point of view of a single core microprocessor, every cache hit is a success. However, from the point of view of a memory-bound many core microprocessor, the goal is to fully utilize of the off-chip memory bandwidth. As the cache hit rate increases, it leaves a reactive cache hierarchy guessing how best to use the available bandwidth and, in some cases, doing nothing. Every unused cycle to off-chip memory is lost and cannot be recovered in the future.

This paper introduces a novel memory subsystem that, when combined with an appropriate computational model, is designed to efficiently utilize the available memory bandwidth while hiding memory latency in highly parallel systems. It creates a queue of outstanding memory requests which allows a pro-active memory subsystem to reorder requests to utilize multiple memory channels in the most efficient ways. To accomplish this, we propose an abstraction that separates the computational load from the memory subsystem via multiple banks of scratchpad memories per computational core, an interconnection network, and the proactive memory management subsystem.

This imposes a few constraints on the computational load. First, it assumes that the application has a large degree of parallelism and that many independent tasks are available. For reasons beyond the scope of this paper, we further assume that these are relatively short, atomic sequences; small bits of imperative programs. The reason for multiple banks is so that compute cores can be oversubscribed. By allowing a core to work out of one memory bank allows another bank to be involved in a memory transaction, resulting in the overlap of computation and communication. This is accomplished by multiplexing the banks of memory and the introduction of a scheduler.

Given these assumptions, the system level view becomes a stochastic process with computational cores providing a statistical demand on the memory subsystem that can be represented by a random variable (an arrival rate) and the memory subsystem responding based on a random variable (a service rate). In such a circumstance, the overall goal of the system is to balance computation against the physical limitations of off-chip memory bandwidth to create a queue of enough outstanding requests that the memory subsystem can intelligently order the transactions. From a formal point of view, it is Little's Law [2, 


$$
N=\lambda \times W
$$

where $N$ is the number of outstanding requests, $\lambda$ is the arrival rate, and $W$ is the wait time. In our case, $\lambda$ (the computational load) can be manipulated so that $N$ does not exceed the on-chip resources. Hence, we create an artificial stationary process that, as the on-chip resources increase, allows us to approach $100 \%$ bandwidth utilization. Combined with the latency-hiding technique introduced in the previous paragraph, this approach has the potential of dramatically improving the overall throughput of highly parallel applications on manycore architectures.

The remainder of this paper describes the first-cut design of the system just outlined. In Section 2, overall (chip level) system is presented along with the details of the proposed memory subsystem. The chip-level design includes a mix of high performance (single thread low latency processor cores) and many high throughput small processor cores. To test the feasibility and practical application of these ideas, a subset of the proposed system has been implemented on an FPGA device. In particular, the ability to connect source and sink of memory segments has been implemented as a B-Tree data structure in hardware. Section 3 describes the physical implementation used to collect the data in the results section, Section 4. A brief summary and description of (the extensive) future work is as described in Section 5 .

\section{Design}

\subsection{Abstract Model}

An abstract view of a generic system design is illustrated in Figure 2 Here, we assume a system containing several different cores, each with some amount of local memory (or scratchpad). There are two types of cores in the system - Green core and White core. The cores are differentiated based on how they are used. The White core is a modern, highly optimized processor core with a traditional cache hierarchy and intended for sequential parts of the application. The Green cores, which are the cores that use the novel memory subsystem proposed here, provide the parallelism. As such, they are either application-specific accelerators or processor cores that favor small size over speed (to increase the maximum degree of parallelism). In a new programming model and run-time system, described in [4, the White core is responsible for generating a tasks that are sent to the Green cores for computation, assigning more than one task per core. Based on the tasks assigned, a Green core will request the code and data by name for all of its tasks simultaneously. Once the memory subsystem has delivered all of the required data for a task to run, the task runs to completion and informs the memory subsystem that a new, named memory segment is available. The central idea is that the memory subsystem can be moving data to the scratchpad memories, and from the scratchpad memories, concurrent with tasks running on the Green cores. When a task completes, the selected scratchpad is changed and the core is reset. Note that under this scheme, there is no global address space, only named memory segments. These memory segments are locally read/write-able 
in an imperative style but once a task completes and the output is shared with the memory subsystem, it is read-only. Communication and synchronization between the cores is handled by references to named segments. Names are ASCII strings that have been hashed to a fixed-width, binary digest, i.e. SHA-like.

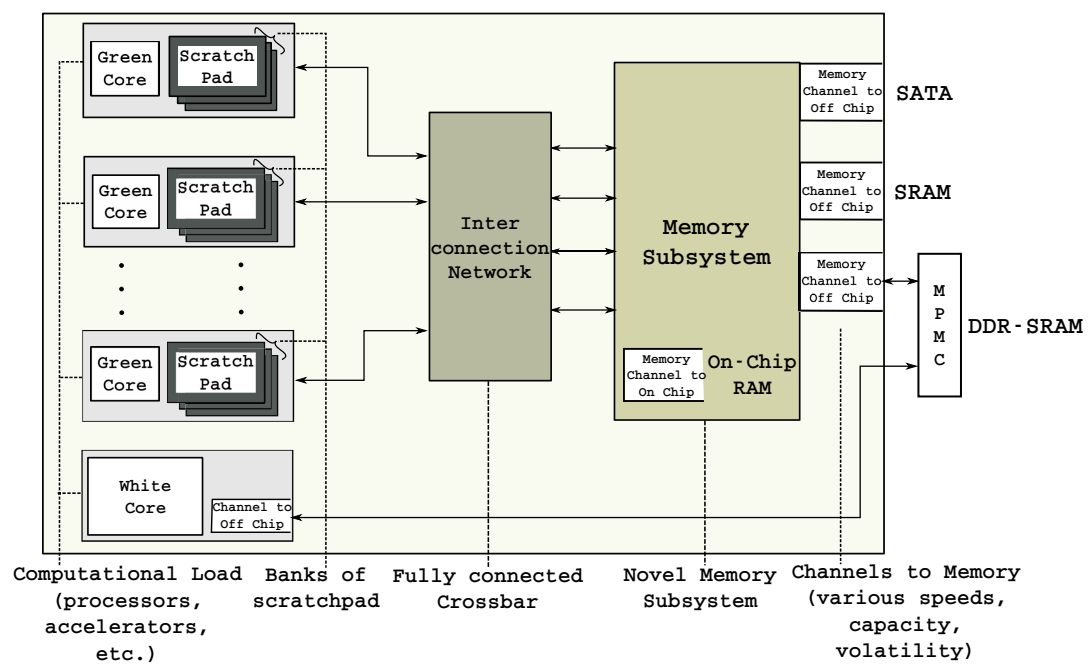

Fig. 2. Many Core Chip Model

This model does not allow for conventional synchronization primitives such as semaphores and it imposes a functional style of programming at the highest level ( [4] describes the two-level programming model in detail). However, it does allow for state machines and imperative style programming at the task level and the organization overcomes several very difficult architectural challenges for extreme scale systems; one of which is the Memory Wall. And finally, as the illustration suggests, there could be multiple channels to memory with different speed, capacity, and volatility characteristics. In this paper, we consider one memory channel to a pool of on-chip blocks of RAM. However, we envision a system with multiple channels to RAM that include external SRAM, DRAM, and SATA.

\subsection{Theory of Operation}

To realize the abstract model of computation presented in this section and utilize the stochastic characteristics, a key operation in the memory subsystem is the ability to dereference memory segment names. This operation takes a name and identifies the location of the memory segment. The segment ideally might exist on chip, in DRAM, or even on a solid state drive. 
A factor in large and complex systems is the storage of metadata that has to be accessed often and quickly. This led to the investigation of data structures implemented in hardware to hold system metadata. Several factors have to be taken into consideration while implementing a data structure in hardware. Highly complex data structures that would be ideal in software, may not fare well in hardware because of the cost. This led us to first investigate the simplest data structure, a binary tree. Previously, a memory system using an ordered binary tree structure was implemented in hardware called the Dynamic Memory Allocation Controller [9. The results definitely proved that it was feasible to implement a data structure in hardware but several issues rendered this structure not optimal for use in hardware.

The next step to our investigation was a B-tree structure in hardware. In this paper, a variation of a B-tree structure implemented in hardware is presented. A B-tree is a multi-way tree in which each node can hold multiple elements called keys. With more than just one key in a node, there can exist multiple children (branches) thereby containing the height of the tree. The B-tree is sorted by the key. The order of the B-tree $(o)$ dictates the number of keys per node. For a $\mathrm{B}$-tree with order $o$, the number of keys each node can have is $o-1$. When there are more than $o-1$ keys, the existing node is split to accommodate the new key. A B-tree, like a binary tree, grows downwards but facilitates re-balancing by splitting a node and adding it to its parent. Compared to the binary tree structure, the B-tree has several advantages. It can support more than just two children per node, which leads to reduced access times. Also the possibility of a bad data pattern leading to a linked list is reduced as the B-tree has a way of balancing out the data within a main branch.

\section{Implementation}

The memory subsystem hardware consists of a master controller, two B-tree modules, and a BRAM pool for data. This hardware serves as an active memory controller, which not only services the requests that are coming in, but can also keep track of pending requests. A unique feature of the memory subsystem is that it can work on variable sized memory chunks. Another feature is that it works on name based memory chunks that make it easy for the processor. For the purpose of this paper, the name is a unique identifier calculated by the master controller. Figure 3 shows a high level block diagram of the memory subsystem.

\subsection{B-Tree Module}

The B-tree module hardware consists of three main components: a B-tree table (BRAM table), a compare-sort module and a controller. This B-tree structure is used to hold the meta data of the system and is implemented in on-chip memory.

B-Tree Memory Table. The table in the BRAM contains the following fields

- key, parent address, left address, right address, node pointer (the base address 


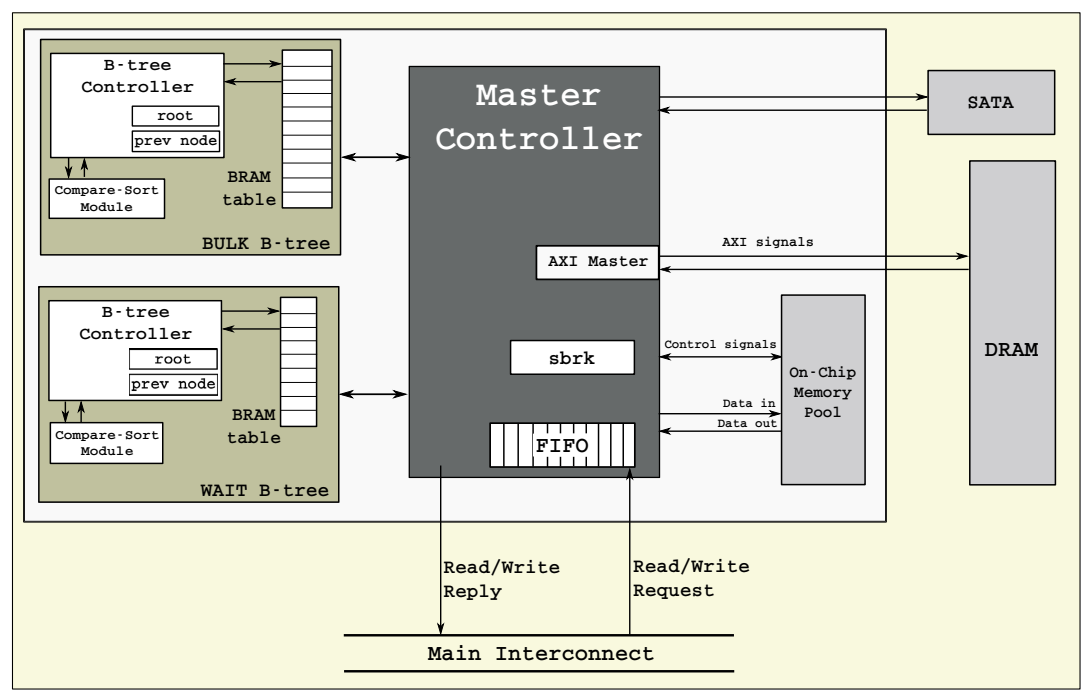

Fig. 3. High level block diagram of the Memory Subsystem

of the data block in memory) and a couple of fields for checking validity and adjacency. Although the B-tree is stored as a table in hardware, in order to achieve the working of a B-tree, it is essential to keep track of the children (branches) and the parent. The size of the key is configurable for this data structure. For the purpose of storing the parent and left/right (branches) the BRAM address of the key which is the parent or left/right branch is captured. The node pointer also has some control bits that indicate the memory location (whether on-chip off-chip) of the data block.

B-Tree Controller. The B-tree controller unit is the top-level hardware that performs all the operations and manages the B-tree table. Keeping in mind the working of a B-tree, multiple consequent splits could result in an intermittent change of the root. The B-tree controller comprises of a root register that keeps track of the current root of the B-tree. The B-tree controller also has four key registers to hold the four keys of the current node in operation. Additionally, the controller retains the latest node that is fetched in a register on-chip. This results in faster look-ups of any keys near a recently referenced key. Every time a request comes in, this register is checked for a match before proceeding to traverse the B-tree.

As in any conventional cache, the B-tree controller receives Read and Write commands. These commands are then interpreted by the B-tree controller as Btree operations and processed accordingly. The B-tree operations include $A d d$, Find, Split, and Update. These commands are explained in detail below:

Add. On receiving a Write command, the B-tree controller adds the particular key to the B-tree table. In doing so, the B-tree controller first performs an 
add operation. Starting at the root, the new key is compared to the existing keys and added to the B-tree accordingly. If it is the first key to be added, it becomes the root and the root register is updated to reflect this key. If a root exists, the key is inserted at an appropriate empty slot in the tree.

Split. While performing an add operation, if the new key is to be inserted at a node that already contains four keys, a split is executed. A split can result in multiple splits, until the B-tree controller finds an appropriate empty slot to insert the new key.

Update. An add or a spilt operation warrants updating of parent/children addresses. A simple add operation would result in updating the parent to reflect the newly added node as its child. A split would result in a more complicated update operation, where the parent and the children have to be updated.

Traverse and Validate. An operation that walks the tree uses these operations. The traverse operation walks the tree, while the validate operation performs checks for the validity of the parent/children.

Find. On receiving a Read command, the B-tree controller issues a find to retrieve the requested key from the B-tree. This command typically traverses the tree looking for the said key. When the key is found, it retrieves the data packet associated with the key and passes it on the memory subsystem.

Delete. For the purpose of this paper, the delete operation only marks the key corresponding to the memory chunk to be invalid. Once the key has been marked for delete, that key is not considered while traversing the B-tree.

Compare-Sort Module. The keys in each B-Tree node require to be sorted in order to achieve precise placement of the children. The input to this module is a node. This module then uses comparators to compare the keys and returns the node with the keys sorted in ascending order. Several operations of the B-Tree require comparing and sorting the keys. The compare-sort module eases this operation, by making it a two-step, feed-in read-out process.

\subsection{BRAM Pool}

While the B-tree module is used to store the meta data of the system, there is an additional resource on-chip to store the actual data. As the name suggests, it is a pool of multiple BRAMs that hold the data. The master controller reads from and writes to the BRAM pool. The size of the BRAM pool is decided based on amount of resources available on-chip.

\subsection{Master Controller}

The master controller is the main pivot that controls the entire system. As shown in Figure 3, the master has control of two B-tree modules - the BULK B-tree module and the WAIT B-tree module. While the basic hardware of the B-tree modules is the same, the main difference is that the WAIT module is used to 
store any request that comes in which does not have any data associated with it yet. Also, since there will never exist data associated with the requests in the WAIT B-tree, this B-tree table does not contain a field to store node pointers. Instead it makes a note of the core that is initiating this request.

For any write request that comes in, the master first checks in the WAIT tree if there has been a request for this data. If there has been a request, the master first services this request by sending the data associated with the request to the core as stored in the WAIT B-tree. Also, in the WAIT B-tree, this entry is now marked for delete. Additionally, this data is written into the $B U L K$ B-tree so any other read requests for this particular data chunk can be serviced accordingly.

The master controller stores the base addresses of the data blocks stored in the BRAMs in the BULK tree. Each request has a payload length associated with it. This controller keeps the BRAM pool in check and allocates and frees BRAMs from the BRAM pool. To ensure that the BRAMs are written to properly, the master controller uses a $\boldsymbol{s} \boldsymbol{b} \boldsymbol{r} \boldsymbol{k}$ register. This register is the hardware equivalent of the sbrk memory management system call in Unix. Every time a new data chunk is stored in the BRAM pool, this register increments by the number of bytes equal to the payload length. Thus, this register always points to the latest available location in the BRAM pool. Additionally, there is a FIFO to queue multiple requests that are pending. The AXI master to external DRAM is a part of the master controller but because of the assumption of a single channel to the memory, this is not explained.

\section{Results}

The design presented has been realized on a Xilinx ML-605 developer board. The Xilinx Embedded development Kit (EDK) version 14.1 was used to develop and synthesize the designs. To test the functionality, a synthetic sequence of message operations (with randomly sized messages) was generated and used to exercise the memory subsystem hardware. The memory subsystem hardware was built as a proof-of-concept system to test the functionality and feasibility of constructing a new approach to memory subsystems using data structures that are implemented in the hardware of the FPGA.

For the initial feasibility test, the size of the key was set to 16 bits. The order of the B-tree is 5 which means there are no more than 4 keys in every node. The size of both the bulk B-tree and the wait B-tree (the BRAM tables) was kept to 1024 nodes. Additionally, size of the BRAM pool was kept to be 4096 . The system when synthesized, utilizes 17037 out of the 150720 slices which is about $11 \%$. The structures implemented were kept to a minimum size in order to gauge the resource utilization. On a Virtex 6 FPGA 12 RAMB36E1s are used exclusively for the memory subsystem hardware. This shows that there is a lot of scope to increase the size of the memory subsystem which will in turn help in observing the theoretical results previously stated.

Table 1 shows the time (in clock cycles) for the basic operations. The depth of the tree, $d$, is an important factor to these results. The Traverse operation is 
Table 1. Clock Cycles for each operation

\begin{tabular}{c|c}
\hline Operation & Cycles per level \\
\hline ADD & 6 \\
FIND & 2 \\
TRAVERSE & $8 \times d$ \\
VALIDATE & $4+d$ \\
\hline
\end{tabular}

directly proportional to the depth of the tree, specifically, $8 \times d$. The Validate operation takes 4 clock cycles and additionally adds one clock cycle per unit increase in depth. This does not apply to the Add and Find operations as the traversal points the controller to the right place, after which only the new key needs to be added, or in the case of Find, returned. The split operation is the most expensive, especially if it results in multiple splits. The time measured for a split is 30 clock cycles. These numbers have been measured in simulation, but the functionality of the memory subsystem has been tested on the FPGA board. In the next version of the system, the idea is to widen the BRAMs to hold an entire node of the B-tree as opposed to just one key. This will help reduce the look up times for the B-tree.

\section{Conclusion}

The memory subsystem and model proposed attempts to hide memory latency and more efficiently use the bandwidth to external memory. It accomplishes this by proactively managing a large number of outstanding requests in such a way that the channel to external memory is fully utilized. To be successful, it imposes a task-oriented computational model on computational cores and requires them to multi-task in order to generate multiple, overlapping memory requests that can be served by the memory subsystem in any order. Using Little's Law, this paper shows that such an organization creates a stochastic system at the architecture level that hides memory latency, increases the effective bandwidth of an off-chip memory channel, and increases the task completion rate for memorybound systems. To demonstrate the feasibility of such a memory subsystem, a hardware based B-tree implementation of a proactive cache has been implemented and tested on a Virtex 6 FPGA.

This approach is not appropriate for all settings. In particular, many FPGAbased designs have an enormous degree of parallelism but are not memory bound. It may be the case that there are designs that interface to instruments, sensors and actuators, that provide a continuous source or sink of information. In other cases there are applications that are very computationally intensive and have a relatively small state. Nonetheless, there is a large range of applications in the High-Performance Computing domain (from graph algorithms to iterative algorithms) that fit the proposed model. 
In the future, a priority scheme will be implemented to allow the memory subsystem to re-order the tasks to always keep the cores busy with computation. Additionally, this design will be extended to include a Least-Recently Used capability that tracks on-chip blocks in the BRAM pool. This will enable the memory subsystem to handle a wide range of memory technologies that vary in capacity, bandwidth, and latency. For example, it could extend memory subsystem to include non-volatile Flash devices as well as DDR synchronous DRAM, and static RAM.

\section{References}

1. Albonesi, D.: Selective cache ways: on-demand cache resource allocation. In: Proceedings of the 32nd Annual International Symposium on Microarchitecture, MICRO-32, pp. 248-259 (1999)

2. Bertsimas, D., Nakazato, D.: The distributional little's law and its applications. Operations Research 43(2), 298-310 (1995)

3. Frigo, M., Johnson, S.: Fftw: an adaptive software architecture for the fft. In: Proceedings of the 1998 IEEE International Conference on Acoustics, Speech and Signal Processing, vol. 3, pp. 1381-1384 (1998)

4. Huang, B., Sass, R., DeBardeleben, N., Blanchard, S.: Pydac: A resilient runtime framework for divide-and-conquer applications on a heterogeneous many-core architecture. In: The 6th Workshop on UnConventional High Performance Computing, UCHPC at Euro-Par 2013 (2013)

5. Johnson, J.R.: Automated performance tuning. In: Proceedings of the 4th International Workshop on Parallel and Symbolic Computation, PASCO 2010, pp. 20-21. ACM, New York (2010), http://doi.acm.org/10.1145/1837210.1837215

6. Kozyrakis, C., Patterson, D.: A new direction for computer architecture research. Computer 31(11), 24-32 (1998)

7. Njoroge, N., Casper, J., Wee, S., Teslyar, Y., Ge, D., Kozyrakis, C., Olukotun, K.: Atlas: a chip-multiprocessor with transactional memory support. In: Proceedings of the Conference on Design, Automation and Test in Europe, DATE 2007, pp. 3-8, EDA Consortium, San Jose (2007), http://dl.acm.org/citation. cfm?id=1266366.1266370

8. Kogge, P., et al.: Exascale computing study: Technology challenges in achieving exascale systems. Tech. Rep. TR-2008-13, DARPA Information Processing Techniques Office (IPTO) sponsored study (2008),

http://www.cse.nd.edu/Reports/2008TR-2008-13.pdf

9. Rajasekhar, Y., Sass, R.: A first analysis of a dynamic memory allocation controller (dmac) core. In: Proceedings of the 2011 Symposium on Application Accelerators in High-Performance Computing, SAAHPC 2011, pp. 64-67. IEEE Computer Society, Washington, DC (2011), http://dx.doi.org/10.1109/SAAHPC.2011.23

10. Wulf, W.A., McKee, S.A.: Hitting the memory wall: implications of the obvious. SIGARCH Comput. Archit. News 23(1), 20-24 (1995), http://doi.acm.org/10.1145/216585.216588 